\title{
Influence of Long-range Interactions on the Critical Behavior of Systems with negative Fisher-Exponent
}

\author{
H.K. JANSSEN \\ Institut für Theoretische Physik III, Heinrich-Heine-Universität, \\ 40225 Düsseldorf, Germany
}

(May 13, 2021)

\begin{abstract}
The influence of long-range interactions decaying in $d$ dimensions as $1 / R^{d+\sigma}$ on the critical behavior of systems with Fisher's correlation-function exponent for short-range interactions $\eta_{S R}<0$, is re-examined. Such systems, typically described by $\Phi^{3}$-field theories, are e.g. the Potts-model in the percolation-limit, the Edwards-Anderson spin-glass, and the Yang-Lee edge singularity. In contrast to preceding studies, it is shown by means of Wilson's momentum-shell renormalization-group recursion relations that the long-range interaction dominates as long as $\sigma<2-\eta_{S R}$. Exponents change continuously to their short-range values at the boundary of this region.
\end{abstract}

PACS-numbers: 64.60.Ak, 05.40.+j, 64.60.Fr

Fifteen years ago there was some debate about the influence of long-range interactions (decaying as $J(R) \sim$ $1 / R^{d+\sigma}$ in $d$ dimensions) on the critical exponents of systems that show a negative Fisher-exponent $\eta_{S R}$ if the long-range forces are absent [1],2]. Finally it was claimed that the long-range interactions leading to a Fisher-exponent $\eta_{L R}=2-\sigma$ dominate for all $\sigma<2$, and, by reason of an instability, the exponents change discontinuously to their short-range values at $\sigma=2$. The assumption that long-range interactions decaying with $\sigma>2$ are generally equivalent to purely short-range interactions seems to be the accepted lore. Indeed in the Gaussian part of an effective Landau-Ginzburg-Wilson Hamiltonian in momentum space $\mathcal{H}_{0}=\frac{1}{2} \int_{q}\left(r+j_{2} q^{2}+j_{\sigma} q^{\sigma}+\ldots\right) s_{q} s_{-q}\left(s_{q}\right.$ is the Fourier transform of an order parameter fluctuation and $\int_{q} \ldots=\int d^{d} q \ldots$ ) where as usual $j_{2}$ stems from the short-range part of the interaction (which also contributes to $r$ ) and $j_{\sigma}$ from the long-range one, $j_{\sigma}$ is naively irrelevant in comparison to $j_{2}$ at long-wave lengths if $\sigma>2$. Of course, this is an incorrect argumentation below the upper critical dimension where the nonquadratic higher-order terms of the Hamiltonian play a dominant role. The relevance of the long-range term $\propto q^{\sigma}$ (which does not renormalize because of its nonanalyticity in $q$ ) has to be determined by comparison with the scaling behavior of the full inverse correlation function $\Gamma_{2}(q)_{S R} \propto q^{2-\eta_{S R}}$ that corresponds to the nontrivial stable fixed point solution of a properly chosen renormalization group transformation.

In the present letter I show that also in the case $\eta_{S R}<0$ the long-range interaction dominates as long as $2-\sigma=\eta_{L R}>\eta_{S R}$. At $\eta_{L R}=\eta_{S R}$ the exponents change continuously to their short-range values that hold everywhere for $\sigma>2-\eta_{S R}$. This behavior is well known for models with $\eta_{S R}>0$ [3] but is seemingly nonaccepted as yet for $\eta_{S R}<0$. The incorrect result of [1, 2] with respect to the crossover arises from a renormalization group which is not appropriate for that case because it mixes in a redundant operator in the terminology of Wegner [8]. I show that in general the limit $\sigma \rightarrow 2$ produces a contribution $\sim q^{2} \ln q$ to the Gaussian part of the Hamiltonian that is a relevant perturbation here. Thus the critical exponents are different for the cases with or without such a perturbation that is therefore responsible for the apparent discontinuity of exponents.

Systems with a negative Fisher exponent $\eta_{S R}$ are typically described by critical $\Phi^{3}$-field theoretic models [9] with an upper critical dimension $d_{c}=6$ such as the Potts model in the percolation limit $[10-12]$, the YangLee singularity model 13, 14], and the Edwards-Anderson spin-glass model in the replica formalism 15.16. Besides these equilibrium models, nonequilibrium models of $\Phi^{3}$ type are given by epidemic processes which lead to percolation clusters. Here long-range interactions creep in if the disease spreads by Levy-flights [17,18]. Treating these nonequilibrium models I became aware of the earlier work [1,2] on long-range interactions in $\Phi^{3}$-models.

In this letter I concentrate on the discussion of the Yang-Lee model as the simplest among all others leading to similar recursion equations. To stick close to the above mentioned work, I use the Wilson renormalization group transformation based on the elimination of short-wave fluctuations and a hard momentum cutoff normalized to $q_{c}=1$. I perform the renormalization group to one-loop order and use $\varepsilon$-expansion where $\varepsilon=6-d$. This is sufficient to produce the nontrivial crossover behavior, since $\eta_{S R}=O(\varepsilon)$.

I write the Yang-Lee-Hamiltonian of the scalar field $s$ in the following form

$$
\begin{gathered}
\mathcal{H}=\int d^{d} x\left\{\frac{1}{2}(\nabla s)^{2}+\frac{v}{4 \alpha}\left[\left(\nabla^{1-\alpha} s\right)^{2}-(\nabla s)^{2}\right]\right. \\
\left.+\frac{r}{2} s^{2}+\frac{i g}{6} s^{3}+i h s\right\} .
\end{gathered}
$$

Here $\left(\nabla^{1-\alpha} s\right)^{2}$ is defined in momentum space as $q^{2(1-\alpha)} s_{q} s_{-q}$, and the momentum scale is chosen such that $|q| \leq q_{c}=1$. I have written the long-range exponent 
as $\sigma=2(1-\alpha)$. Then $\alpha=O(\varepsilon)$ in the crossover region. The Gaussian part of $\mathcal{H}$ with the derivatives of the field is positively definite as long as $v \geq 0$ irrespective of the sign of $\alpha$, and reads in momentum space $\frac{1}{2} q^{2}(1-v \ln q) s_{q} s_{-q}$ in the limit $\alpha \rightarrow 0$. Thus also in this limit the model does not coincide with its short-ranged counterpart unless $v=O(\alpha)$. The unperturbed correlation function (the propagator of a diagrammatic perturbation expansion) follows from (1) as

$$
G_{0}(q)=\left(q^{2}+\frac{v}{2 \alpha}\left[q^{-2 \alpha}-1\right] q^{2}+r\right)^{-1} .
$$

Thus the scale of the fields is defined such that for $r=0$ the propagator is 1 at the cut-off momentum $q_{c}=1$. The propagator is positively definite for all $q$ and $\alpha$ as it should be for stability.

The calculation of the momentum integrals that arise by eliminating to one-loop order fluctuations $s_{q}$ which depend on momenta in the interval $b^{-1}<q \leq 1$ with $b \approx 1$ is standard and does not present any technical difficulties. The coefficients of the terms of different order in the Hamiltonian $\mathcal{H}$ (11) change by the elimination procedure to

$$
\begin{aligned}
& q^{2}\left\{1+\frac{v}{2 \alpha}\left(q^{-2 \alpha}-1\right)\right\}+r \\
\rightarrow & q^{2}\left\{1+\frac{v}{2 \alpha}\left(q^{-2 \alpha}-1\right)-\frac{2 u K(v, r)}{d(1+r)^{4}} \ln b\right\} \\
+ & \left\{r+\frac{u}{(1+r)^{2}} \ln b\right\}+O\left(q^{4}, u^{2}\right)
\end{aligned}
$$

and

$$
\begin{aligned}
& g \rightarrow g\left\{1-\frac{2 u}{(1+r)^{3}} \ln b+O\left(q^{2}, u^{2}\right)\right\} \\
& h \rightarrow h+\frac{u / g}{1+r} \ln b .
\end{aligned}
$$

Here, I have defined $u=(4 \pi)^{-d / 2} g^{2} / \Gamma(d / 2)$, and $K(v, r)$ is given by

$$
\begin{aligned}
K(v, r)= & \frac{d-2}{4}+\frac{2+2 \alpha-d}{8} v-\frac{v^{2}}{8} \\
& -\frac{1-\alpha}{4} v r+\frac{2-v}{8} d r
\end{aligned}
$$

After elimination of the short-wavelength fluctuations an appropriate rescaling has to be introduced as the last step of the renormalization transformation. The goal is to choose a rescaling $\zeta$ of the fields $s^{<}(\mathbf{r})=\zeta s^{\prime}\left(b^{-1} \mathbf{r}\right)$ where $s^{<}(\mathbf{r})=\int_{q \leq b^{-1}} e^{i \mathbf{q r}} s_{\mathbf{q}}$ in such a way that a renormalization group is constructed which leads to fixed points and does not mix in redundant dangerous operators [8]. The operator generated by a rescaling of the fields without an elimination is such a redundant operator. Thus the rescaling factor $\zeta$ must be chosen carefully.
The old (and working) definition for $\zeta$ follows from the requirement to hold the propagator finite at the cutoff momentum to exclude infrared singularities. Then such singularities cannot arise in the full elimination procedure. Note that in the long-range interaction problem normally one holds constant the coefficient of the nonanalytic term $\sim q^{2(1-\alpha)}$. This may be the simplest possibility but leads to the difficulty of a vanishing inverse propagator in the present case [1,2]. Therefore I define

$$
\zeta^{2}=b^{2-d-\gamma(u, v)} .
$$

Here the function $\gamma(u, v)$ follows from the requirement $G_{0}^{\prime}\left(q=1, r^{\prime}=0\right)=G_{0}(q=1, r=0)=1$ for the rescaled propagator. Now by rescaling (3, A), renormalized parameters are found from

$$
\begin{aligned}
& q^{2}\left\{1+\frac{v^{\prime}}{2 \alpha}\left(q^{-2 \alpha}-1\right)\right\} \\
= & q^{2} b^{-\gamma}\left\{1+\frac{v}{2 \alpha}\left(b^{2 \alpha} q^{-2 \alpha}-1\right)-\frac{2 u K(v, r)}{d(1+r)^{4}} \ln b\right\},
\end{aligned}
$$

and

$$
\begin{aligned}
& r^{\prime}=b^{2-\gamma}\left\{r+\frac{u}{(1+r)^{2}} \ln b\right\}, \\
& u^{\prime}=u b^{6-d-3 \gamma}\left\{1-\frac{4 u}{(1+r)^{3}} \ln b\right\}, \\
& h^{\prime}=b^{(d+2-\gamma) / 2}\left\{h+\frac{u / g}{1+r} \ln b\right\} .
\end{aligned}
$$

Taking (8) for $q=1$ yields the equation for $\gamma$ :

$$
1=b^{-\gamma}\left\{1+\frac{v}{2 \alpha}\left(b^{2 \alpha}-1\right)-\frac{2 u K(v, r)}{d(1+r)^{4}} \ln b\right\} .
$$

Expanding $b=\exp l$ to first order in $l$ around $l=0$ one finds

$$
\begin{aligned}
\gamma & =v-\frac{2 u K(v, r)}{d(1+r)^{4}} \\
& =v-\frac{u}{3}\left(1-\frac{2-\alpha}{4} v-\frac{v^{2}}{8}\right)+O\left(\varepsilon^{2}\right) .
\end{aligned}
$$

In the last equation I have used $r=O(u), u=O(\varepsilon)$, and $I$ have retained only terms linear in $\varepsilon$. Note that the dependence on $v$ is exact to linear order in $u$. For an infinitesimal transformation with $l=d l$ one gets the renormalization group equations from the remaining parts of the equations (8,9,10,11) as

$$
\begin{aligned}
& \frac{d u}{d l}=(\varepsilon-3 \gamma-4 u) u, \\
& \frac{d v}{d l}=(2 \alpha-\gamma) v, \\
& \frac{d r}{d l}=(2-\gamma) r+\frac{u}{(1+r)^{2}}, \\
& \frac{d h}{d l}=\frac{d+2-\gamma}{2} h+\frac{u}{g(1+r)} .
\end{aligned}
$$


It follows from the nonanalyticity of the long-range term of the Hamiltonian (11) that $v$ does not acquire any contribution by the elimination step and changes only under the rescaling. Thus the second equation (15) is exact, whereas the other three are correct only to one-loop order.

The last two equations $(16,17$ ) show in general fixed points $r_{*}, h_{*}=O\left(u_{*}\right)$ for the relevant parameters $r$ and $h$. Using these values the first two equations (14, 15) in combination with $\gamma$ (13) lead to four different fixed points for the coupling constants $u$ and $v$. There are two Gaussian ones $\left(u_{*}=0\right)$, namely a short-range fixed point with $v_{*}=0$, stable for $\varepsilon<0, \alpha<0$ with $\eta:=\gamma_{*}=0$, and a long-range fixed point with $v_{*}=\eta=2 \alpha$, stable for $\varepsilon<6 \alpha, \alpha>0$. Beside these trivial fixed points there are two nontrivial ones with $u_{*}>0$. The well-known 13, 14 short-range fixed point with $v_{*}=0$ follows from (14) as $u_{*}=\varepsilon / 3+O\left(\varepsilon^{2}\right)$ and leads to $\eta=\eta_{S R}=-\varepsilon / 9+O\left(\varepsilon^{2}\right)$. It is stable for $\varepsilon>0$ as long as $2 \alpha<\eta_{S R}$. But if $2 \alpha>\eta_{S R}$, it becomes unstable and the long-range fixed point develops from (15) with $v_{*}=2 \alpha+(\varepsilon-6 \alpha) / 12+O\left(\varepsilon^{2}\right), u_{*}=(\varepsilon-6 \alpha) / 4+O\left(\varepsilon^{2}\right)$, and is stable up to $\alpha=\varepsilon / 6$, and $\eta=\eta_{L R}=2 \alpha$. The stability regions are shown in Fig. 1. In each case the shortrange behavior changes continuously to the long-range behavior and vice versa at the line defined by $\eta_{S R}=\eta_{L R}$. For all fixed points, the correlation length exponent $\nu$ follows from the linearized equation (16) for $r-r_{*}$. Here, in the case of the Yang-Lee model, a Ward identity states $\beta=1$ for the order parameter exponent 9, 14 and one obtains $\nu=2 /(d-2+\eta)$ for both nontrivial fixed points.

To get a picture of the renormalization flow of the coupling constants and the movement of the fixed points for $\varepsilon>0$, I have rescaled the variables as $x=u / \varepsilon, y=v / \varepsilon$, and introduced the parameter $p=2 \alpha / \varepsilon$. With a "time" $t=l / \varepsilon$ the equations of motion are found as

$$
\begin{aligned}
& \dot{x}=(1-3 x-3 y) x \\
& \dot{y}=\left(p+\frac{x}{3}-y\right) y .
\end{aligned}
$$

The flows and the fixed points are shown in Fig. 2 for different parameter values. Again one sees the continuous bifurcation of the different fixed points corresponding to the continuous crossover of short-range and long-range behavior.

In conclusion I have shown that an old result concerning the crossover between long- and short-range interaction behavior in critical systems with negative shortrange Fisher exponent $\eta_{S R}$ is incorrect. As in the case of a positive Fisher exponent, the behavior changes continuously at a line defined by $\eta_{S R}=\eta_{L R}=2-\sigma$. The longrange interactions dominate always as long as $\eta_{S R}<\eta_{L R}$.

\section{ACKNOWLEDGMENTS}

I thank S. Theiss for a critical reading of the manuscript. This work has been supported in part by the SFB 237 ("Unordnung und große Fluktuationen") of the Deutsche Forschungsgemeinschaft.

[1] M.-c. Chang and J. Sak, Phys. Rev. B 29, 2652 (1984).

[2] W. K. Theumann and M. A. Gusmão, Phys. Rev. B 31, 379 (1985).

[3] M. E. Fisher, S.-k Ma, and B. G. Nickel, Phys. Rev. Lett. 29, 917 (1972).

[4] J. Sak, Phys. Rev. B 8, 281 (1973).

[5] A. Aharony, in Phase Transitions and Critical Phenomena, Vol. 6, edited by C. Domb and M. S. Green (Academic, New York, 1976).

[6] Y. Yamazaki, Il Nuovo Cimento 55, 59 (1980).

[7] J. Honkonen and M. Yu. Nalimov, J. Phys. A: Math. Gen. 22, 751 (1989).

[8] F. J. Wegner, in Phase Transitions and Critical Phenomena, Vol. 6, edited by C. Domb and M. S. Green (Academic, New York, 1976).

[9] O. F. de Alcantara Bonfim, J. E. Kirkham, and A. J. McKane, J. Phys. A: Math. Gen. 13, L247 (1980); J. Phys. A: Math. Gen. 14, 2391 (1981).

[10] A. B. Harris, T. C. Lubensky, W. K. Holcomb, and C. Dasgupta, Phys. Rev. Lett. 35, 327 (1975).

[11] D. J. Amit, J. Phys. A: Math. Gen. 9, 1441 (1976).

[12] R. G. Priest and T. C. Lubensky, Phys. Rev. B 13, 4159 (1976); Phys. Rev. B 14, 5125(E) (1976).

[13] M. E. Fisher, Phys. Rev. Lett. 40, 1610 (1978).

[14] N. Breuer and H. K. Janssen, Zeitschr. f. Phys.: Cond. Mat. B 41, 55 (1981).

[15] S. F. Edwards and P. W. Anderson, J. Phys. F: Metal Phys. 5, 965 (1975).

[16] A. B. Harris, T. C. Lubensky, and J.-H. Chen, Phys. Rev. Lett. 36, 415 (1976).

[17] P. Grassberger, in Fractals in Physics, edited by L. Pietronero and E. Tosatti (Elsevier, 1986).

[18] H. K. Janssen, to be published.

[19] G. Kotliar, P. W. Anderson, and D. L. Stein, Phys. Rev. B 27, 602 (1983).

[20] C. N. Yang and T. D. Lee, Phys. Rev. 87, 404 (1952).

FIG. 1. Renormalization flow of the coupling constants $x=u / \varepsilon, y=v / \varepsilon$ for different parameter values $p=2 \alpha / \varepsilon$ in the case of $\varepsilon=6-d>0$ to one-loop order. The topology of the flow changes continuously with $p$, and there exists always a stable long-range $(y>0)$ fixed point if $p>-1 / 9$.

FIG. 2. The stability regions of long- and short-range behavior. Nontrivial short- (SR) and long-range (LR), as well as trivial Gaussian short- (GSR) and Gaussian long-range (GLR) regions are shown. The behavior changes continuously at the boundaries. 


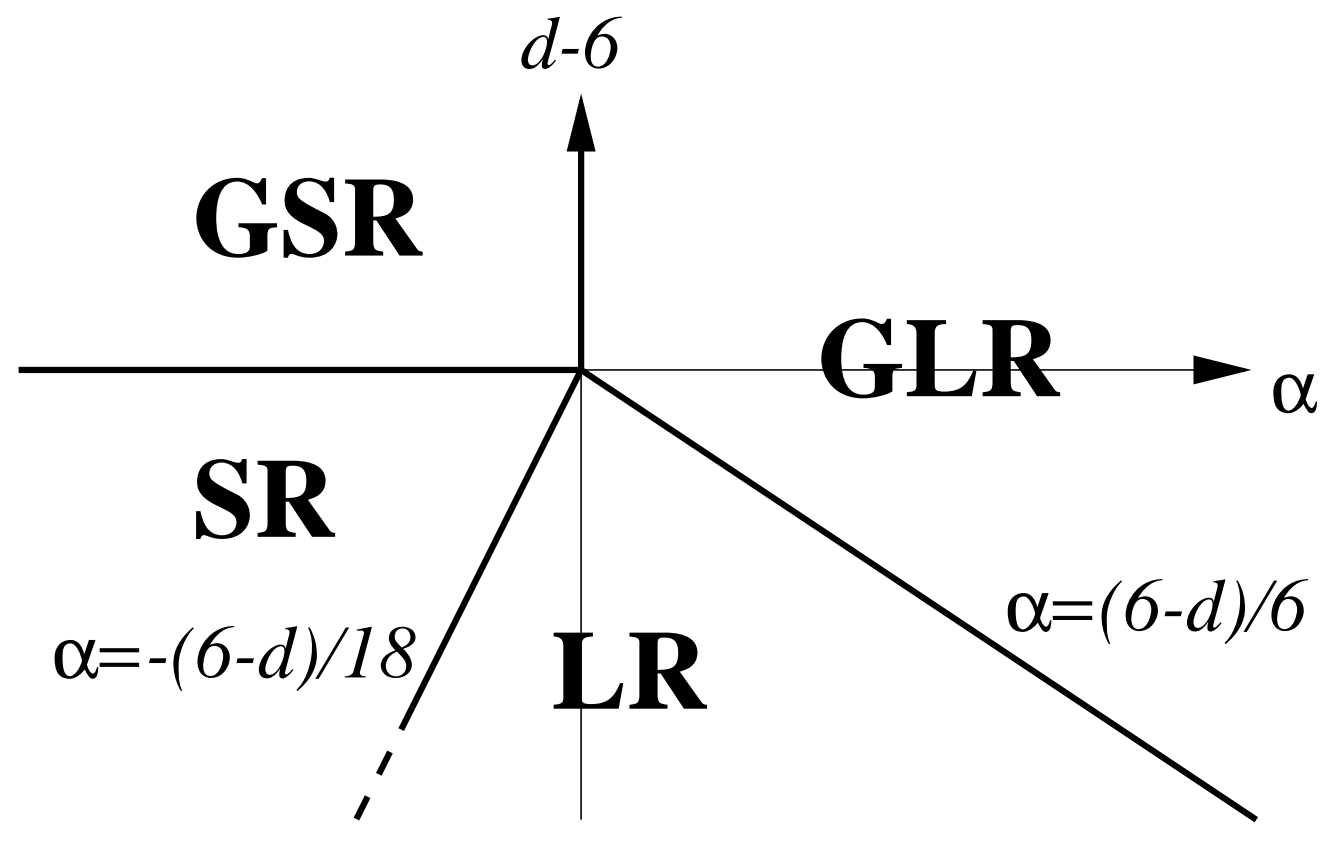



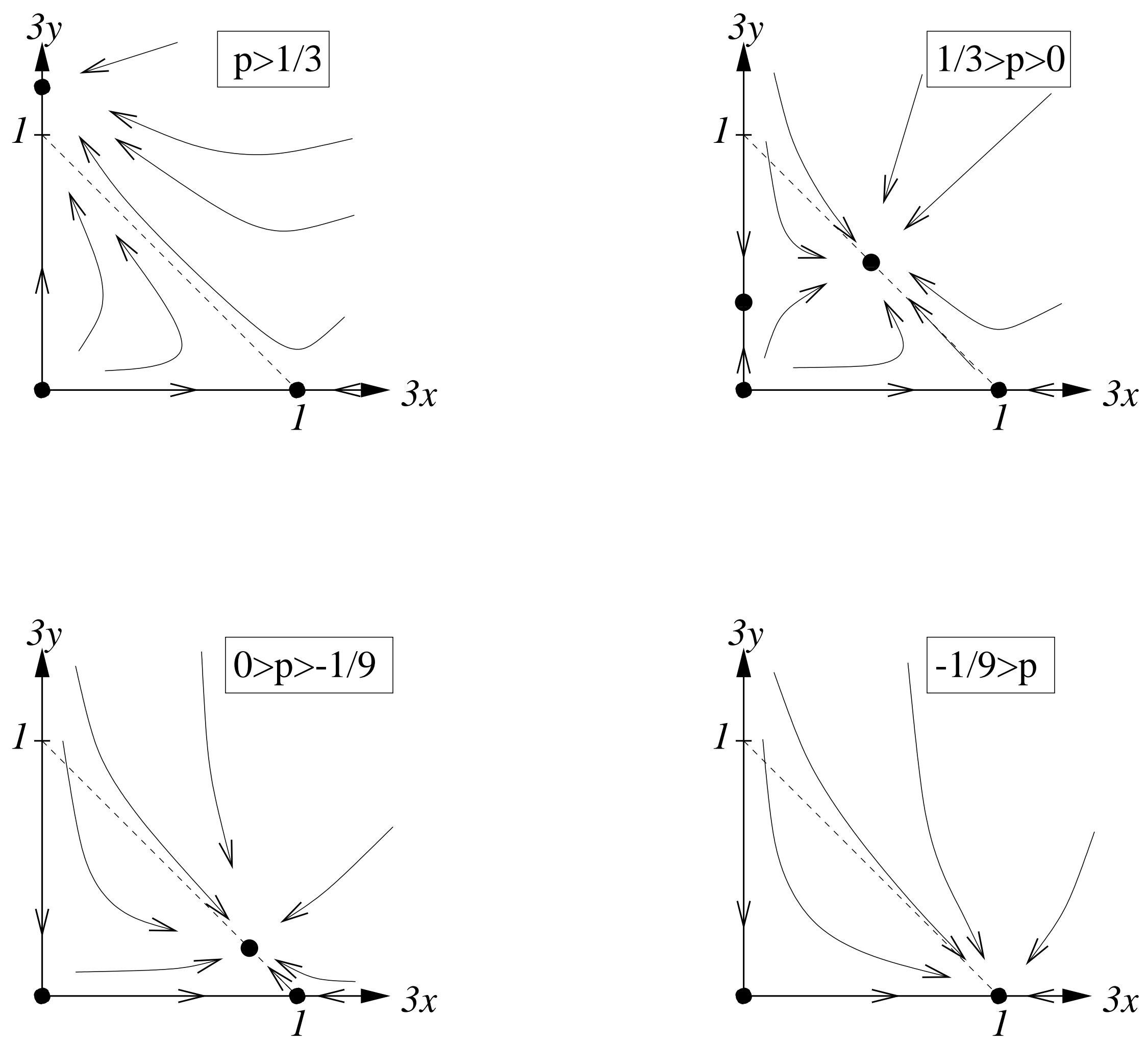